\title{
Second Harmonic Generation of Violet Light in Femtosecond-Laser-Inscribed $\mathrm{BiB}_{3} \mathrm{O}_{6}$ Cladding Waveguides
}

\author{
Yuechen Jia ${ }^{1}$, Javier R. Vázquez de Aldana ${ }^{2}$, Qingming $\mathrm{Lu}^{3}$, Daniel Jaque ${ }^{4}$, and Feng Chen ${ }^{1}$ \\ ${ }^{I}$ School of Physics, State Key Laboratory of Crystal Materials and Key Laboratory of Particle Physics and Particle Irradiation (MOE), \\ Shandong University, Jinan 250100, China \\ ${ }^{2}$ Laser Microprocessing Group, Universidad de Salamanca, Salamanca 37008, Spain \\ ${ }^{3}$ School of Chemistry and Chemical Engineering, Shandong University, Jinan 250100, China \\ ${ }^{4}$ Departamento Física de Materiales, Universidad Autónoma de Madrid, Madrid 28049, Spain \\ E-mail:jiayuechen0521@163.com,drfchen@sdu.edu.cn
}

\begin{abstract}
We report on the second harmonic generation of violet light of a nonlinear cladding waveguide in $\mathrm{BiB}_{3} \mathrm{O}_{6}$ crystal produced by femtosecond laser inscription. Under continuous-wave pump laser at $800 \mathrm{~nm}$, the guided second harmonic wave at $400 \mathrm{~nm}$ with a conversion efficiency of $\sim 0.32 \%$ has been realized through the Type I birefringence phase matching configuration.
\end{abstract}

\section{Introduction}

Optical waveguides are desired in photonics owing to the inside high optical intensities with respect to bulk materials. High-performance photonic devices could be constructed and integrated in small circuits by using waveguide structures [1]. Since 1996, the femtosecond (fs) laser inscription has emerged to be a powerful technique to fabricate waveguides for its unique ability on the three-dimensional (3D) micromachining of many transparent materials [2-4]. Depressed cladding waveguides are confined in the regions surrounded with relatively high refractive index damage lines or filament tracks induced by the fs-lasers. Such a configuration was firstly proposed by Okhrimchuk et al [5]. One of the most intriguing advantages of cladding waveguides is that the cross section could be designed with circular geometry and its diameter could match that of the multimode fibers (fiber-like cross section), which is very promising for the construction of fiber-waveguide-fiber integrated photonic chips. In addition, the cladding waveguides may support guidance along two transverse polarizations, which is particularly important for nonlinear frequency doubling.

Bismuth borate $\left(\mathrm{BiB}_{3} \mathrm{O}_{6}\right)$ is a very attractive nonlinear crystal with large nonlinear optical coefficients and a high damage threshold. The second harmonic generation (SHG) from visible till UV light band can be achieved in $\mathrm{BiB}_{3} \mathrm{O}_{6}$ via birefringent phase matching $(\mathrm{PM})$. We have reported the fabrication of cladding waveguides in this crystal and realized the $1064 \rightarrow 532 \mathrm{~nm}$ SHG [6]. In this work, we report on the SHG features of the $\mathrm{BiB}_{3} \mathrm{O}_{6}$ cladding waveguide for $800 \rightarrow 400 \mathrm{~nm}$ SHG of violet light.

\section{Experiments and results}

The optically polished $\mathrm{BiB}_{3} \mathrm{O}_{6}$ crystal wafer was prepared to fit the direction of the $800 \rightarrow 400 \mathrm{~nm}$ birefringent PM SHG in the $y$-z plane $\left(\theta=151.2^{\circ}, \varphi=90^{\circ}\right)$. The cladding structure was produced by using fs-laser-inscription (details on the experiment can be found in [6]). The nonlinear performance of the waveguide was characterized by utilizing a typical end-face coupling system. A CW Ti:sapphire laser (Coherent MBR 110) generating a linearly polarized beam at $\sim 800 \mathrm{~nm}$ was employed as a pump source. A convex lens with a focal length of $25 \mathrm{~mm}$ was used to couple the fundamental wave into the waveguide. The generated SH wave was collected by a $20 \times$ microscope objective. The FD was realized by using a TE-polarized $800 \mathrm{~nm}$ pump, generating SH along TM polarization, i.e., under $\mathrm{TE}^{\omega}+\mathrm{TE}^{\omega} \rightarrow \mathrm{TM}^{2 \omega}$, which is in accordance with the bulk Type I PM $(e+e \rightarrow o)$.
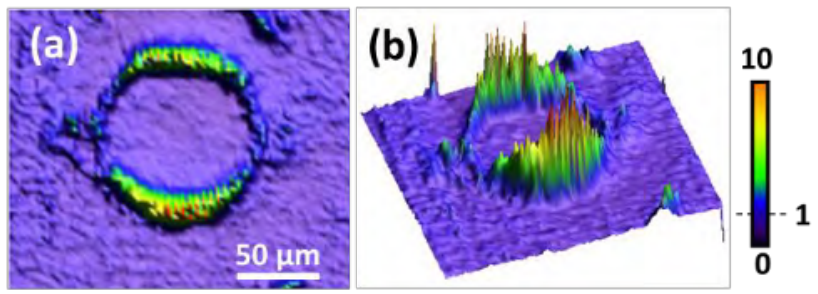

Fig. 1. (a) $2 \mathrm{D}$ and (b) $3 \mathrm{D}$ confocal $\mu$-SH cross-sectional mappings obtained from the end face of the $\mathrm{BiB}_{3} \mathrm{O}_{6}$ cladding waveguide.

In order to investigate the preservation, at the waveguide volume, of the original nonlinear properties after fs laser inscription, we have measured the $2 \mathrm{D}$ and $3 \mathrm{D}$ confocal $\mu$-SH mappings of the end face of $\mathrm{BiB}_{3} \mathrm{O}_{6}$ cladding 
waveguide from 800 to $400 \mathrm{~nm} \mathrm{SHG} \mathrm{(as} \mathrm{shown} \mathrm{in} \mathrm{Fig.} \mathrm{1).} \mathrm{It} \mathrm{can} \mathrm{be} \mathrm{observed} \mathrm{that} \mathrm{the} \mathrm{nonlinear} \mathrm{response} \mathrm{of} \mathrm{the}$ $\mathrm{BiB}_{3} \mathrm{O}_{6}$ crystal has not been modified in the waveguide volume, suggesting effective preservation of the nonlinear properties within the waveguide volume. As a matter of fact, the SH response has been found to be modified only at the waveguide boundary, and this relevant SH enhancement taking place has been attributed to an enhancement in the back-reflection efficiency due to the presence of defects acting as scattering centers.

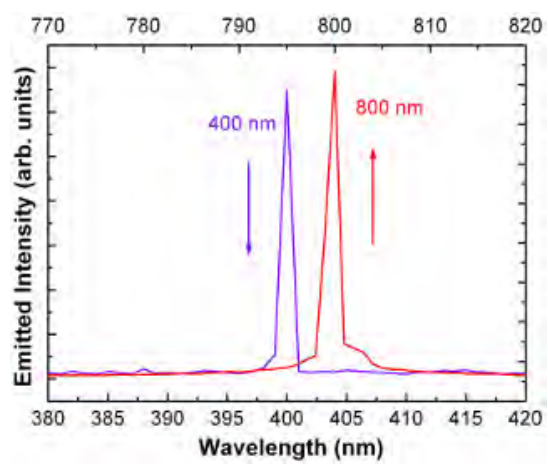

Fig. 2. Laser spectra of the fundamental (red) and $\mathrm{SH}$ (violet) waves from the $\mathrm{BiB}_{3} \mathrm{O}_{6}$ cladding waveguide.

Figure 2 depicts the typical spectra of the fundamental $800 \mathrm{~nm}$ and SH $400 \mathrm{~nm}$ waves from the waveguide under CW pump. It should be noted that for the cladding waveguide, the laser spectra for fundamental and $\mathrm{SH}$ waves are similar to those from the bulk, respectively, which also suggests that the nonlinear properties of the crystal have been well preserved in the waveguide structures.

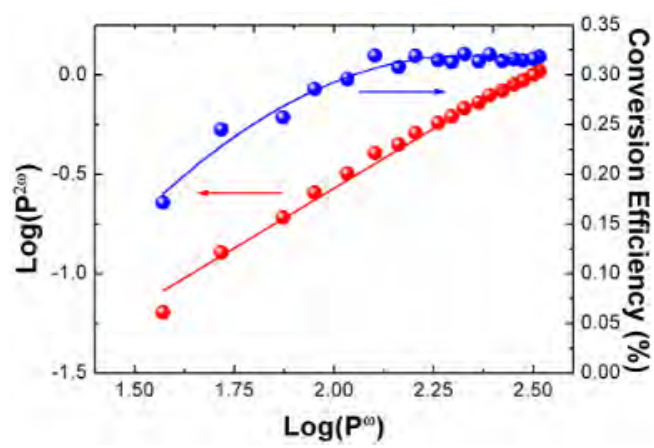

Fig. 3. SHG output power and conversion efficiency versus the fundamental pump power of the cladding waveguides in $\mathrm{BiB}_{3} \mathrm{O}_{6}$ crystal. The solid lines represent the fit of the experimental data.

Figure 3 shows the power of the output SH wave at $400 \mathrm{~nm}$ and SH conversion efficiency as a function of that of the $800 \mathrm{~nm}$ fundamental wave from the cladding waveguides. We obtained a maximum output power of approximately $\sim 1.05 \mathrm{~mW}$ under pump of $\sim 328 \mathrm{~mW} 800-\mathrm{nm}$ light, which corresponds to an SH conversion efficiency of $\eta=\sim 0.32 \%$ (normalized value of $\sim 0.98 \% / \mathrm{W}$ ). The SHG performance indicates potential applications of the fs-laser-inscribed $\mathrm{BiB}_{3} \mathrm{O}_{6}$ cladding waveguides as efficient integrated frequency converters.

In summary, the SHG has been successfully achieved for the $800 \rightarrow 400 \mathrm{~nm}$ conversion though the $\mathrm{BiB}_{3} \mathrm{O}_{6}$ cladding waveguide fabricated by fs-laser-inscription. Confocal $\mu$-SH mappings have revealed that the nonlinear responses of $\mathrm{BiB}_{3} \mathrm{O}_{6}$ have been well preserved within the waveguide volume, and we have obtained up to $\sim 1.05$ $\mathrm{mW}$ with a conversion efficiency of $\sim 0.32 \%$ at $400 \mathrm{~nm}$ when the waveguide was optically excited at $800 \mathrm{~nm}$.

\section{References}

[1] E. J. Murphy, Integrated optical circuits and components: Design and applications (Marcel Dekker, 1999).

[2] R. R. Gattass and E. Mazur, "Femtosecond laser micromachining in transparent materials," Nat. Photonics 2, 219-225 (2008).

[3] S. Juodkazis, V. Mizeikis, and H. Misawa, "Three-dimensional microfabrication of materials by femtosecond lasers for photonics applications,” J. Appl. Phys. 106, 051101 (2009).

[4] K. M. Davis, K. Miura, N. Sugimoto, and K. Hirao, "Writing waveguides in glass with a femtosecond laser," Opt. Lett. 21, 1729-1731 (1996).

[5] A. G. Okhrimchuk, A. V. Shestakov, I. Khrushchev, and J. Mitchell, "Depressed cladding, buried waveguide laser formed in a YAG:Nd ${ }^{3+}$ crystal by femtosecond laser writing," Opt. Lett. 30, 2248-2250 (2005).

[6] Y. C. Jia, J. R. Vazquez de Aldana, C. Romero, Y. Y. Ren, Q. M. Lu, and F. Chen, "Femtosecond-Laser-Inscribed BiB $\mathrm{O}_{6} \mathrm{Nonlinear}^{-}$

Cladding Waveguide for Second-Harmonic Generation," Appl. Phys. Express 5, 072701 (2012). 